\title{
QUALIDADE FÍSICA DE UM LATOSSOLO VERMELHO ACRIFÉRRICO SOB DIFERENTES SISTEMAS DE USO E MANEJO(1)
}

\author{
Ricardo Garcia Aratani ${ }^{(2)}$, Onã da Silva Freddi ${ }^{(3)}$, José Frederico \\ Centurion $^{(4)} \&$ Itamar Andrioli $^{(5)}$
}

\begin{abstract}
RESUMO
Os sistemas de manejo podem promover a degradação da qualidade física do solo, com reflexos ambientais e na produtividade. Nesse sentido, o objetivo deste trabalho foi avaliar e comparar propriedades relacionadas à qualidade física de um Latossolo Vermelho acriférrico de Guaíra, Estado de São Paulo, entre os tratamentos: sistema plantio direto irrigado, sistema plantio direto de sequeiro, integração lavoura-pecuária, plantio convencional e mata natural. Foram avaliados o conteúdo de matéria orgânica, a estabilidade de agregados, a densidade do solo, a porosidade e o índice $\mathrm{S}$. Os resultados mostraram que a ação antrópica reduziu a qualidade do solo e o sistema plantio direto não aumentou o teor de matéria orgânica, porém apresentou maiores índices de agregação do que o plantio convencional. O tempo de adoção do sistema plantio direto, aliado à irrigação, aumentou a estabilidade dos agregados em todas as camadas e o volume de macroporos na camada 0-0,10 m. A integração lavoura-pecuária não melhorou a qualidade física do solo, e o índice $\mathrm{S}$ mostrou variação em função dos sistemas e foi sempre superior ao valor considerado para boa qualidade física do solo.
\end{abstract}

Termos de indexação: sistema plantio direto, física do solo, estabilidade de agregados.

\footnotetext{
(1) Parte da Tese de Doutorado do primeiro autor. Trabalho financiado pela FAPESP. Recebido para publicação em março de 2008 e aprovado em março de 2009.

(2) Doutorando em Agronomia, Ciência do Solo. Universidade Estadual Paulista - UNESP. Campus de Jaboticabal. Via de Acesso Prof. Paulo Donato Castellane s/n, CEP 14884-900 Jaboticabal (SP). Bolsista FAPESP. E-mail: aratani@fcav.unesp.br

(3) Pós-doutorando, Universidade Estadual Paulista - UNESP. Faculdade de Ciências Agrárias e Veterinárias. Departamento de Solos e Adubos. Bolsista FAPESP. E-mail: ona_freddi@yahoo.com.br

(4) Professor Adjunto. Universidade Estadual Paulista - UNESP. Faculdade de Ciências Agrárias e Veterinárias. Departamento de Solos e Adubos. Bolsista CNPq. E-mail: jfcentur@fcav.unesp.br

${ }^{(5)}$ Professor Adjunto. Universidade Estadual Paulista - UNESP. Faculdade de Ciências Agrárias e Veterinárias. Departamento de Solos e Adubos. E-mail: solos@fcav.unesp.br
} 


\title{
SUMMARY: PHYSICAL QUALITY OF AN ANIONIC ACRUSTOX UNDER DIFFERENT MANAGEMENT SYSTEMS
}

\begin{abstract}
Agricultural management systems can cause degradation of the soil physical quality, with consequences to the environment and crop yields. Thus, the purpose of this study was to evaluate and compare the physical quality of an anionic Acrustox in Guaira, São Paulo state, under the following treatments: no-tillage with irrigation, no-tillage without irrigation, croppasture rotation, conventional tillage, and native forest. The organic matter content, aggregate stability, soil bulk density, porosity and index $S$ were evaluated. Results showed that human intervention reduced the soil quality and that no-tillage led to no increase in organic matter contents, but it did increase aggregation indices as compared with conventional tillage. In the no-tillage system plus irrigation, the aggregate stability increased in all evaluated soil layers and there also was gains in macropore volume in the 0-0.10 $\mathrm{m}$ soil layer over time. The use of crop-pasture rotation did not improve the soil physical quality and the index $S$ varied according to the systems, although it was always higher than the value suggested as limit for good soil physical quality.
\end{abstract}

Index terms: no-tillage system, Physical characteristics, aggregates stability.

\section{INTRODUÇÃO}

A avaliação da qualidade do solo não é uma ideia nova, no entanto, recentemente alguns autores começaram a desenvolver métodos para essa atividade, definindo-a concisamente como o grau de aptidão do solo para um uso específico (Doran et al., 1996; Carter et al., 1997; Gregorich, 2002). Assim, o tipo de solo, o clima e as culturas envolvidas nos diferentes sistemas de manejo podem promover a degradação da qualidade física do solo, com reflexos ambientais e na produtividade das culturas (Tormena et al., 2004).

Usualmente, a qualidade do solo agrícola é considerada sobre três aspectos: físico, químico e biológico, sendo importantes nas avaliações da extensão da degradação ou melhoria do solo e para identificar a sustentabilidade dos sistemas de manejo. No entanto, a qualidade física do solo merece destaque especial em novos estudos, pois acredita-se que tem grande efeito nos processos químicos e biológicos no solo, porém pouco explorado nos estudos de qualidade do solo (Dexter, 2004a). Klepker \& Anghinoni (1995) afirmam que, quando a qualidade física do solo é prejudicada pelo preparo, a distribuição e a morfologia das raízes são afetadas, com reflexos no crescimento da parte aérea, principalmente devido às altas densidades do solo e à resistência à penetração e à baixa porosidade.

$\mathrm{O}$ uso de métodos que quantifiquem e qualifiquem as condições estruturais do solo nos vários sistemas de manejo é importante na avaliação da qualidade do solo, considerada um indicador da sustentabilidade dos sistemas de uso e manejo (Arshad et al., 1996). Assim, os impactos do uso e manejo na qualidade física do solo têm sido quantificados, utilizando diferentes propriedades físicas relacionadas com sua forma e com sua estabilidade estrutural, como a estabilidade de agregados, a densidade e a porosidade do solo. O índice
"S" introduzido no Brasil por Silva (2004), a partir dos trabalhos de Dexter (2004a,b,c), calculado a partir da curva de retenção de água, apresenta importante contribuição para os estudos da qualidade do solo, principalmente pelo grande potencial para ser usado em avaliações da interação entre o manejo e a qualidade física e ser facilmente medido, usando equipamentos e equações utilizados rotineiramente nos laboratórios de física do solo.

Um índice quantitativo de qualidade do solo pode servir como indicador de sua capacidade para a produção sustentável de plantas e de animais de forma econômica, social e ambientalmente aceitáveis.

O objetivo deste trabalho foi avaliar e comparar as propriedades relacionadas à qualidade física no que diz respeito ao manejo de um Latossolo Vermelho acriférrico submetido a quatro sistemas de uso, em mata nativa, sob plantio direto e convencional.

\section{MATERIAL E MÉTODOS}

\section{Local e solo}

O estudo foi realizado em talhões de lavouras comerciais, sob um Latossolo Vermelho acriférrico típico textura argilosa (Embrapa, 2006), situado no município de Guaíra, norte do Estado de São Paulo ( $20^{\circ} 21^{\prime} \mathrm{S}, 48^{\circ} 15^{\prime} \mathrm{W}$ e $551 \mathrm{~m}$ de altitude). O município, localizado na região da Alta Mogiana, segundo a classificação de Köppen, apresenta clima de inverno seco (Cwa), com temperatura média anual de $24^{\circ} \mathrm{C}$ e $1.300 \mathrm{~mm}$ de precipitação pluvial, distribuídos entre outubro e abril. Os conteúdos médios de areia, silte e argila, na camada de $0-0,20 \mathrm{~m}$ das áreas avaliadas foram: 184, 61 e $755 \mathrm{~g} \mathrm{~kg}^{-1}$, respectivamente. 


\section{Sistemas de manejo}

Os manejos avaliados foram: plantio direto irrigado de 12 anos - PD12; plantio direto sequeiro de 5 anos PD5; plantio direto sequeiro de 5 anos, com integração lavoura-pecuária a 2 anos-PD5I; plantio convencional $-\mathrm{PC}$ e mata natural $(\mathrm{MN})$.

A área com o sistema PD12 apresenta sistema de irrigação por pivô central e utiliza a rotação de milho com a soja no verão a cada três anos. No outonoinverno, rotacionam o milho, o sorgo e o feijão. O sistema PD5 tem o milho substituindo a soja no verão a cada três anos e o milho safrinha ou o sorgo no outonoinverno. A área no sistema PD5I iniciou o sistema plantio direto juntamente com a área no sistema PD5, diferindo na incorporação da integração com a pecuária, utilizando o Sistema Santa Fé. Nesse sistema, a braquiária para a formação de pasto é semeada juntamente com a adubação de cobertura do milho. A planta de milho se desenvolve primeiro, ficando a braquiária com seu crescimento prejudicado, devido ao sombreamento. Com a maturação e o secamento do milho, a braquiária então continua seu crescimento, ganhando tempo e condições favoráveis para a formação do pasto (Embrapa, 2000). O Sistema PC é o plantio convencional em que o preparo do solo utiliza uma gradagem pesada e duas passadas de grade niveladora, há mais de vinte anos. $\mathrm{Na}$ safra avaliada, o agricultor optou pela não utilização da gradagem pesada, efetuando apenas uma passada de grade niveladora para posterior semeadura.

\section{Indicadores e coletas das amostras}

As coletas de amostras de solo para a estabilidade de agregados foram realizadas em dezembro de 2005 , nas camadas de $0-0,10 ; 0,10-0,20 ; 0,20-0,30 \mathrm{~m}$, com quatro repetições, com auxílio de um enxadão. No preparo das amostras, os torrões foram secos ao ar e desmanchados manualmente, tomando-se cuidado para não destruir os agregados. Para avaliar a estabilidade de agregados via úmida, foram usados $50 \mathrm{~g}$ de solo passados em peneira de 7,93 $\mathrm{mm}$ e ficaram retidos na peneira de $4,00 \mathrm{~mm}$, os quais foram préumedecidos, conforme o princípio de umedecimento lento descrito por Kemper \& Chepil (1965). Em seguida, essa amostra foi colocada no aparelho de oscilação vertical sobre um conjunto de peneiras de 4,$00 ; 2,00 ; 1,00 ; 0,50 ; 0,25$ e $0,125 \mathrm{~mm}$ de diâmetro de abertura de malha, conforme descrito por Yooder (1936). Transcorridos $15 \mathrm{~min}$, as porções retidas em cada peneira foram transferidas para latas de alumínio com o auxílio de jatos de água, e secas em estufa a $105{ }^{\circ} \mathrm{C}$, por $24 \mathrm{~h}$, para posterior pesagem. A partir dos valores dessas massas, foram calculados a porcentagem de agregados estáveis em água com diâmetro maior que 2,00 mm (PAE), o diâmetro médio geométrico (DMG) e o diâmetro médio ponderado (DMP), conforme Kemper \& Chepil (1965). Para verificar se os valores de DMP dos tratamentos com solo cultivado (PD12, PD5, PD5I e PC) foram diferentes daqueles da MN, calculou-se o índice de sensibilidade (IS) sugerido por Bolinder et al. (1999) e utilizado por Bertol et al. (2004), que utiliza o princípio da comparação relativa entre tratamentos. $\mathrm{O}$ valor do referido índice maior do que a unidade significa que a estabilidade dos agregados aumentou e, quando menor, diminuiu. O teor de matéria orgânica (MO) foi avaliada segundo método descrito por Raij et al. (1987).

Para a determinação das propriedades físicas do solo, foram coletadas amostras com estrutura indeformada em quatro pontos aleatórios para cada sistema de manejo, utilizando cilindros de $52,35 \mathrm{~cm}^{3}$, no ponto médio das profundidades de $0-0,10,0,10-0,20$ e 0,20 $0,30 \mathrm{~m}$. Foram determinadas a densidade do solo (Blake \& Hartge, 1986) e a microporosidade por secagem (tensão de 0,006 MPa) em câmaras de pressão de Richards com placa porosa (Klute, 1986), a porosidade total segundo Danielson \& Sutherland (1986). A macroporosidade foi obtida por diferença entre a porosidade total e a microporosidade. As amostras indeformadas também foram submetidas às tensões de 0,$06 ; 0,01 ; 0,033 ; 0,06,0,1$ e 0,3 MPa, em câmaras de Richards (Klute, 1986), para obtenção das curvas de retenção de água do solo em cada camada e manejo, utilizando-se o modelo proposto por van Genuchten (1980), de onde foram retirados os parâmetros de ajuste utilizados no cálculo do Índice S.

No cálculo do índice S, o foco principal está na inclinação da curva de retenção de água do solo no ponto de inflexão. Isto pode ser medido diretamente por meio da curva de retenção; entretanto, é mais conveniente ajustá-la a uma função matemática e então calcular a inclinação no seu ponto de inflexão (Equação 1), utilizando os parâmetros obtidos na equação de van Genuchten (1980). Segundo Dexter (2004a), S é igual a:

$$
S=-n\left(\theta_{\text {sat }}-\theta_{\text {res }}\right)\left[1+\frac{1}{m}\right]^{-(1+m)}
$$

em que $n$ e $m$ são parâmetros que governam o formato da curva; $\theta_{\text {sat }}$ e $\theta_{\text {res }}$ são a umidade na saturação e umidade residual, respectivamente.

A condutividade hidráulica saturada (Ksat) foi medida no campo empregando-se duas cargas hidráulicas, 0,03 e 0,06 m, em dezembro de 2005, com quatro repetições, utilizando-se um permeâmetro tipo Guelph (Reynolds et al., 1992).

A resistência do solo à penetração $(\mathrm{RP})$ foi determinada na entrelinha da cultura da soja, utilizando um penetrômetro de impacto modelo IAA/ PLANALSUCAR, desenvolvido por Stolf et al. (1983), em seis repetições por tratamento. Cada repetição foi formada pela média de seis avaliações. Concomitantemente à determinação de RP, foi avaliada a umidade 
do solo pelo método gravimétrico em seis pontos selecionados aleatoriamente ao longo da linha de amostragem de RP.

\section{Análise estatística}

A análise de variância seguiu o delineamento inteiramente casualizado com parcelas subdivididas $5 \times 3$ (cinco manejos e três profundidades), com quatro repetições. Os graus de liberdade para tratamento (manejo) foram decompostos em quatro contrastes ortogonais entre si, dentro de cada camada, para isolar os efeitos do cultivo do solo, do plantio direto, da idade de adoção do plantio direto/irrigação e da integração lavoura-pecuária (Quadro 1). Foi utilizado o teste de Tukey $(p<0,05)$ para comparar as camadas dentro de cada tratamento.

Em virtude do grande número de variáveis, da necessidade de melhorar a compreensão dos fatores que influenciam a qualidade do solo e melhorar a exatidão da descriminação entre os sistemas avaliados, foram aplicados os princípios da estatística multivariada por meio da técnica dos componentes principais para identificar os fatores de alteração associados a cada sistema de manejo. Nesta análise, a variância contida em cada componente principal é expressa pelos autovalores da matriz padronizada. $\mathrm{O}$ maior autovalor é associado ao primeiro componente principal, o segundo maior autovalor ao segundo componente principal, até que o menor autovalor esteja associado ao último componente principal, o que coloca os primeiros como os mais importantes. Sendo assim, os primeiros componentes principais explicam geralmente grande parte da variância das variáveis originais (Agreg, DMP, DMG, índice S, Macro, Micro, Porosidade, Kfs e RP). Esta análise permitiu agrupar as variáveis originais em um conjunto menor, preservando o máximo da variabilidade original dos dados. A adequação desta análise é verificada pela quantidade da informação total das variáveis originais retida pelos componentes principais que possuem autovalores superiores à unidade (Kaiser, 1958). Autovalores inferiores à unidade não possuem informação relevante. A análise estatística multivariada foi processada no software STATISTICA versão 7.0 (Statsoft, 2004).

\section{RESULTADOS E DISCUSSÃO}

\section{Matéria orgânica e estabilidade de agregados}

O cultivo do solo reduziu o conteúdo de matéria orgânica (MO) em todas as camadas (Figura 1). A substituição da mata nativa por culturas anuais causa essa redução em função do revolvimento do solo, o qual favorece a oxidação da MO (Corazza et al., 1999). O sistema plantio direto não foi eficiente em aumentar o conteúdo de MO; pelo contrário, mostrou-se inferior nas camadas de $0-0,10$ e 0,20-0,30 m. Wendling et al. (2005), estudando o efeito de sistemas de manejo em Latossolo Vermelho, observaram que, apesar de o sistema plantio direto aumentar o aporte de fitomassa sobre o solo, o conteúdo de C orgânico total apresentou tendência de diminuição nas camadas superficiais. $\mathrm{O}$

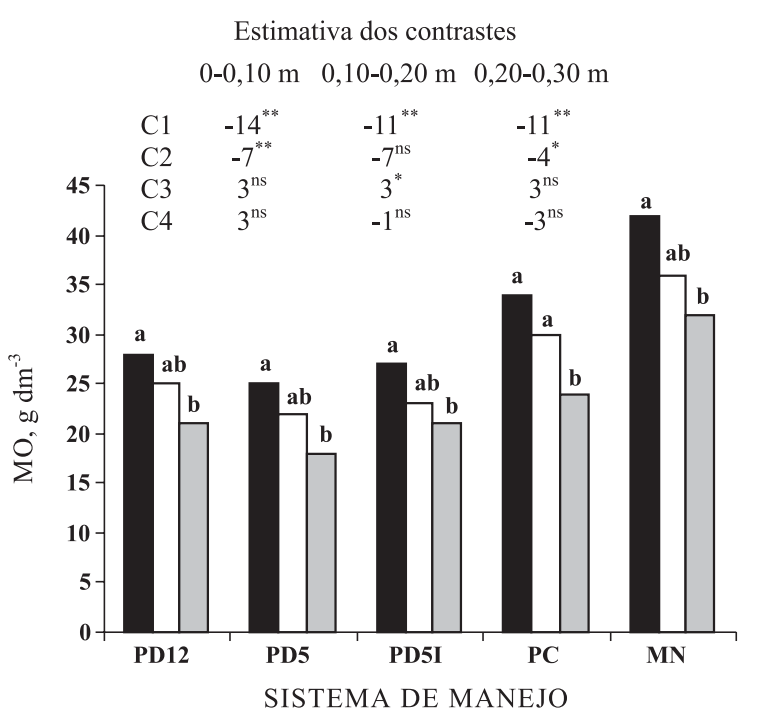

Figura 1. Teores de matéria orgânica (MO) e estimativa dos contrastes estabelecidos para os cinco tratamentos e três profundidades. Camada

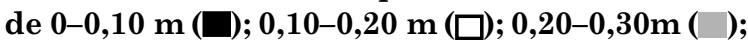
ns: não significativo; ${ }^{* *}$ : significativo a $1 \%$. Médias seguidas pela mesma letra, dentro de cada manejo, não diferem estatisticamente entre si pelo teste de Tukey $(p<0,05)$.

Quadro 1. Nomes, coeficientes e efeitos dos contrastes entre os tratamentos

\begin{tabular}{ccccccl}
\hline \multirow{2}{*}{ Nome } & \multicolumn{3}{c}{ Tratamento $^{(1)}$} & Efeito \\
\cline { 2 - 5 } & PD12 & PD5 & PD5I & PC & MN & Cultivo \\
C1 & 1 & 1 & 1 & 1 & -4 & Plantio direto \\
C2 & 1 & 1 & 1 & -3 & 0 & Idade/ irrigação \\
C3 & 1 & -1 & 0 & 0 & 0 & Integração \\
C4 & 0 & 1 & -1 & 0 & 0 & \\
\hline
\end{tabular}

(1) PD12: plantio direto irrigado há 12 anos; PD5: plantio direto de sequeiro há 5 anos; PD5I: plantio direto de sequeiro há 5 anos, com integração lavoura-pecuária há 2 anos; PC: preparo convencional há mais de 20 anos; MN: mata nativa. 
plantio direto sob irrigação, aliado ao maior tempo de adoção do sistema aumentou, significativamente, o conteúdo de MO na camada $0,10-0,20 \mathrm{~m}$. Não houve efeito da utilização da integração lavoura-pecuária no conteúdo de MO.

O manejo do solo, assim como os teores de MO, proporcionou redução dos índices de agregação em todas as camadas avaliadas, comprovando a perda de estabilidade dos agregados devido ao uso agrícola, em relação à mata nativa (Quadro 2). A maior agregação no sistema MN é resultante do acúmulo de MO ao longo dos anos, em virtude da não ação antrópica por mais de 40 anos, influenciando fortemente a agregação do solo. O mesmo comportamento manifestou-se quando se comparou o sistema plantio direto com o plantio convencional, mostrando o efeito positivo do primeiro. $\mathrm{O}$ efeito positivo do sistema plantio direto nos índices de agregação do solo também foi observado por Wendling et al. (2005), mostrando a importância do menor revolvimento do solo para aumentar a resistência aos processos erosivos, em detrimento do revolvimento excessivo muitas vezes praticado no plantio convencional, com consequente redução do conteúdo de $\mathrm{MO}$, importante agente de formação e estabilização dos agregados (Castro Filho et al., 1998).

O tempo de adoção do sistema plantio direto aliado ao uso de irrigação acarretou melhoria na agregação do solo, evidenciada na camada de $0-0,10 \mathrm{~m}$ pela maior percentagem de agregados com diâmetro $>$ que 2,00 $\mathrm{mm}$ (Agreg), na camada 0,10-0,20 m, efeito positivo em todos os índices de agregação e na camada 0,20-0,30 m, pelo aumento na Agreg, DMG e DMP.

Não houve efeito da utilização da integração lavoura-pecuária nos índices de agregação. Era de se esperar que este contraste fosse significativo, pois

Quadro 2. Percentagem de agregados $>$ 2,00 mm estáveis em água (Agreg), diâmetro médio geométrico (DMG), diâmetro médio ponderado (DMP), índice de sensibilidade (IS) e teste F para os contrastes estabelecidos para cada camada

\begin{tabular}{|c|c|c|c|c|}
\hline \multirow{2}{*}{ Tratamento ${ }^{(2)}$} & \multirow{2}{*}{ Agreg } & \multicolumn{2}{|c|}{ Índice de agregação ${ }^{(1)}$} & \multirow{2}{*}{ IS } \\
\hline & & DMG & DMP & \\
\hline & $\%$ & \multicolumn{2}{|c|}{$\mathrm{mm}$} & \\
\hline & \multicolumn{4}{|c|}{ Camada de $0-0,10 \mathrm{~m}$} \\
\hline PD12 & $95,73 \mathrm{a}$ & $5,08 \mathrm{a}$ & $5,44 \mathrm{a}$ & $0,93 \mathrm{a}$ \\
\hline PD5 & $92,27 \mathrm{a}$ & $4,84 \mathrm{a}$ & $5,41 \mathrm{a}$ & $0,93 \mathrm{a}$ \\
\hline PD5I & $92,24 \mathrm{a}$ & $4,73 \mathrm{a}$ & $5,28 \mathrm{a}$ & $0,91 \mathrm{a}$ \\
\hline $\mathrm{PC}$ & $70,44 \mathrm{~b}$ & $2,75 \mathrm{~b}$ & $3,96 \mathrm{~b}$ & $0,68 \mathrm{~b}$ \\
\hline MN & $99,04 \mathrm{a}$ & $5,75 \mathrm{a}$ & $5,85 \mathrm{a}$ & $1,00 \mathrm{a}$ \\
\hline Contraste & \multicolumn{4}{|c|}{$p>F$} \\
\hline $\mathrm{C} 1$ & $-11,37^{\star \star}$ & $-1,40^{* *}$ & $-0,83^{* *}$ & $-0,14^{* *}$ \\
\hline $\mathrm{C} 2$ & $22,97^{* *}$ & $0,80^{* *}$ & $1,41^{* *}$ & $0,24^{* *}$ \\
\hline C3 & $3,46^{*}$ & $0,24^{\mathrm{ns}}$ & $0,03^{\mathrm{ns}}$ & $0^{\text {ns }}$ \\
\hline \multirow[t]{2}{*}{$\mathrm{C} 4$} & $0,03^{\mathrm{ns}}$ & $0,11^{\mathrm{ns}}$ & $0,13^{\text {ns }}$ & $0,02^{\text {ns }}$ \\
\hline & \multicolumn{4}{|c|}{ Camada de 0,10-0,20 m } \\
\hline PD12 & $94,81 \mathrm{a}$ & $5,12 \mathrm{a}$ & $5,51 \mathrm{a}$ & $0,95 \mathrm{a}$ \\
\hline PD5 & $89,34 \mathrm{a}$ & $4,43 \mathrm{a}$ & $5,16 \mathrm{a}$ & $0,90 \mathrm{a}$ \\
\hline PD5I & $88,56 \mathrm{a}$ & $4,23 \mathrm{a}$ & $5,00 \mathrm{a}$ & $0,88 a$ \\
\hline $\mathrm{PC}$ & $79,37 \mathrm{a}$ & $3,52 \mathrm{a}$ & $4,61 \mathrm{a}$ & $0,81 \mathrm{a}$ \\
\hline $\mathrm{MN}$ & 98,91 a & $5,67 \mathrm{a}$ & $5,79 \mathrm{a}$ & $1,00 \mathrm{a}$ \\
\hline Contraste & & & & \\
\hline $\mathrm{C} 1$ & $-10,89^{* *}$ & $-1,35^{* *}$ & $-0,72^{* *}$ & $-0,12^{* *}$ \\
\hline $\mathrm{C} 2$ & $11,53^{* *}$ & $1,07^{* \star}$ & $0,61^{* *}$ & $0,10^{* *}$ \\
\hline C3 & $5,47^{* *}$ & $0,69^{* *}$ & $0,35^{* *}$ & $0,05^{*}$ \\
\hline \multirow[t]{2}{*}{$\mathrm{C} 4$} & $0,78^{\mathrm{ns}}$ & $0,20^{\mathrm{ns}}$ & $0,16^{\mathrm{ns}}$ & $0,02^{\text {ns }}$ \\
\hline & \multicolumn{4}{|c|}{ Camada de $0,20-0,30 \mathrm{~m}$} \\
\hline PD12 & $97,46 \mathrm{a}$ & $5,36 \mathrm{a}$ & $5,61 \mathrm{a}$ & $0,96 \mathrm{a}$ \\
\hline PD5 & 90,39 a & $4,64 \mathrm{a}$ & $5,26 \mathrm{a}$ & $0,90 \mathrm{a}$ \\
\hline PD5I & $89,59 \mathrm{a}$ & $4,29 \mathrm{a}$ & $5,02 \mathrm{a}$ & $0,85 \mathrm{a}$ \\
\hline $\mathrm{PC}$ & $66,46 \mathrm{~b}$ & $2,51 \mathrm{~b}$ & $3,75 \mathrm{~b}$ & $0,64 \mathrm{~b}$ \\
\hline $\mathrm{MN}$ & 99,15 a & $5,75 \mathrm{a}$ & $5,84 \mathrm{a}$ & $1,00 \mathrm{a}$ \\
\hline Contraste & \multicolumn{4}{|c|}{$p>F$} \\
\hline C1 & $-13,18^{* *}$ & $-1,55^{\star *}$ & $-0,93^{* *}$ & $-0,16^{* *}$ \\
\hline $\mathrm{C} 2$ & $26,02^{* *}$ & $2,25^{\text {** }}$ & $-0,54^{* *}$ & $0,26^{* *}$ \\
\hline C3 & $7,07^{\text {** }}$ & $0,72^{* *}$ & $0,35^{*}$ & $0,06^{*}$ \\
\hline $\mathrm{C} 4$ & $0,80^{\text {ns }}$ & $0,35^{\text {ns }}$ & $0,24^{\mathrm{ns}}$ & $0,05^{\mathrm{ns}}$ \\
\hline
\end{tabular}

(1) Médias seguidas pela mesma letra na coluna e entre as camadas não diferem estatisticamente entre si pelo teste de Tukey $(\mathrm{p}<0,05) .{ }^{(2)} \mathrm{ns}$ : não-significativo; *: significativo a $5 \% \mathrm{e}{ }^{* *}$ : significativo a $1 \%$. 
alguns trabalhos mostram que sistemas de rotação de culturas sob sistema plantio direto envolvendo espécies com sistema radicular agressivo, como o da braquiária e com diferentes quantidades de fitomassa, podem alterar as propriedades físicas do solo (Albuquerque et al., 2001). Provavelmente, o curto tempo de adoção do sistema não permitiu a expressão das características positivas.

A comparação dos atributos avaliados nas diferentes camadas mostrou redução dos índices de agregação no PC nas camadas 0-0,10 e 0,20-0,30 m, em comparação com a camada de $0,10-0,20 \mathrm{~m}$. É possível que a camada $0,10-0,20 \mathrm{~m}$ tenha implementado a preparo do solo, que causa compressão das partículas do solo, formando torrões, sem, entretanto, ocorrerem os mecanismos de formação e estabilização destes, que não apresentam qualidades positivas de um agregado (Silva et al., 2000).

\section{Densidade, porosidade total, macroporosidade e microporosidade do solo}

Os resultados mostram que todos os sistemas de manejo avaliados aumentaram a densidade do solo em relação à mata natural Os maiores valores de macroporos e porosidade total e menores valores de microporos foram conferidos à área de mata natural, na mesma comparação. Esta situação é comum, já que a ação antrópica está relacionada com a compactação do solo pelo uso de máquinas e implementos (Cavenage et al., 1999), com a redução dos teores de MO (Silva \& Kay, 1996) e com a menor estabilidade estrutural do solo (Horn et al., 1995).

Quando comparado ao plantio convencional, verificou-se que o sistema plantio direto apresentou os maiores valores de porosidade total e microporosidade em todas as camadas avaliadas.

Quadro 3. Densidade do solo (Ds), porosidade total, macroporosidade e microporosidade e teste F para os contrastes estabelecidos para cada camada

\begin{tabular}{|c|c|c|c|c|}
\hline \multirow[b]{2}{*}{ Tratamento ${ }^{(2)}$} & \multicolumn{4}{|c|}{ Propriedade $^{(1)}$} \\
\hline & Densidade & Porosidade & Macroporosidade & Microporosidade \\
\hline & \multirow[t]{2}{*}{$\mathrm{Mg} \mathrm{m}^{-3}$} & & \multirow{2}{*}{$\overline{0,10 \mathrm{~m}} \mathrm{~m}^{3} \mathrm{~m}^{-3}$} & - \\
\hline \multicolumn{3}{|c|}{ Camada de $0-0,10 \mathrm{~m}$} & & \\
\hline PD12 & $1,14 \mathrm{a}$ & $0,575 \mathrm{a}$ & $0,087 \mathrm{a}$ & $0,488 \mathrm{a}$ \\
\hline PD5 & $1,17 \mathrm{a}$ & $0,592 \mathrm{a}$ & $0,120 \mathrm{a}$ & $0,472 \mathrm{a}$ \\
\hline PD5I & $1,12 \mathrm{a}$ & $0,590 \mathrm{a}$ & $0,092 \mathrm{a}$ & $0,498 \mathrm{a}$ \\
\hline $\mathrm{PC}$ & $0,92 \mathrm{~b}$ & $0,561 \mathrm{a}$ & $0,168 \mathrm{a}$ & $0,393 \mathrm{~b}$ \\
\hline $\mathrm{MN}$ & $0,78 \mathrm{a}$ & $0,637 \mathrm{a}$ & $0,301 \mathrm{a}$ & $0,336 \mathrm{a}$ \\
\hline Contraste & \multicolumn{4}{|c|}{$p>F$} \\
\hline $\mathrm{C} 1$ & $0,31^{* *}$ & $-0,058^{* *}$ & $-0,184^{* *}$ & $0,127^{* *}$ \\
\hline $\mathrm{C} 2$ & $0,22^{\text {** }}$ & $0,025^{*}$ & $-0,068^{* *}$ & $0,093^{* *}$ \\
\hline C3 & $-0,03 \mathrm{~ns}$ & $-0,017 \mathrm{~ns}$ & $-0,033^{*}$ & $0,016 \mathrm{~ns}$ \\
\hline \multirow[t]{2}{*}{$\mathrm{C} 4$} & $0,05^{\mathrm{ns}}$ & 0,002 ns & $0,028 \mathrm{~ns}$ & $-0,026 \mathrm{~ns}$ \\
\hline & \multicolumn{4}{|c|}{ Camada de $0,10-0,20 \mathrm{~m}$} \\
\hline $\mathrm{PD} 12$ & $1,26 \mathrm{a}$ & $0,564 \mathrm{a}$ & $0,068 \mathrm{a}$ & 0,496 a \\
\hline PD5 & $1,24 \mathrm{a}$ & $0,561 \mathrm{a}$ & $0,076 \mathrm{a}$ & $0,485 \mathrm{a}$ \\
\hline PD5I & $1,15 \mathrm{a}$ & $0,611 \mathrm{a}$ & $0,117 \mathrm{a}$ & $0,494 \mathrm{a}$ \\
\hline $\mathrm{PC}$ & $1,18 \mathrm{a}$ & $0,535 \mathrm{a}$ & $0,082 \mathrm{~b}$ & $0,453 \mathrm{a}$ \\
\hline MN & $0,81 \mathrm{a}$ & $0,602 \mathrm{a}$ & $0,260 \mathrm{a}$ & $0,342 \mathrm{a}$ \\
\hline Contraste & & & $\mathrm{F}$ & \\
\hline $\mathrm{C} 1$ & $0,40^{* *}$ & $-0,034^{* *}$ & $-0,174^{* *}$ & 0,140 ** \\
\hline $\mathrm{C} 2$ & $0,04 \mathrm{~ns}$ & $0,044^{* *}$ & $0,005 \mathrm{~ns}$ & $0,039^{\text {** }}$ \\
\hline C3 & $0,02 \mathrm{~ns}$ & $0,003^{\mathrm{ns}}$ & $-0,008$ ns & 0,011 ns \\
\hline \multirow[t]{2}{*}{$\mathrm{C} 4$} & 0,09 ns & $-0,050$ ** & $-0,041$ ** & $0,009 \mathrm{~ns}$ \\
\hline & \multicolumn{4}{|c|}{ Camada de 0,20-0,30 m } \\
\hline & $1,25 \mathrm{a}$ & $0,565 \mathrm{a}$ & $0,091 \mathrm{a}$ & $0,474 \mathrm{a}$ \\
\hline PD5 & $1,20 \mathrm{a}$ & $0,565 \mathrm{a}$ & $0,104 \mathrm{a}$ & $0,461 \mathrm{a}$ \\
\hline PD5I & $1,15 \mathrm{a}$ & $0,590 a$ & $0,092 \mathrm{a}$ & $0,498 \mathrm{a}$ \\
\hline PC & $1,12 \mathrm{a}$ & $0,544 \mathrm{a}$ & $0,085 \mathrm{~b}$ & $0,459 \mathrm{a}$ \\
\hline $\mathrm{MN}$ & $0,79 \mathrm{a}$ & $0,619 \mathrm{a}$ & $0,285 \mathrm{a}$ & $0,334 \mathrm{a}$ \\
\hline Contraste & \multicolumn{4}{|c|}{$\mathrm{p}>\mathrm{F}$} \\
\hline & $0,39^{\text {** }}$ & & $-0,192^{* *}$ & \\
\hline $\mathrm{C} 2$ & $0,08^{\text {** }}$ & $0,029^{* *}$ & $0,011 \mathrm{~ns}$ & 0,019 * \\
\hline C3 & $0,05 \mathrm{~ns}$ & $0^{\text {ns }}$ & $-0,013 \mathrm{~ns}$ & $0,013 \mathrm{~ns}$ \\
\hline $\mathrm{C} 4$ & 0,05 ns & $-0,025 \mathrm{~ns}$ & $0,012 \mathrm{~ns}$ & $-0,037 *$ \\
\hline
\end{tabular}

(1) Médias seguidas pela mesma letra na coluna e entre as camadas não diferem estatisticamente entre si pelo teste de Tukey $(\mathrm{p}<0,05) .{ }^{(2)}$ ns: não-significativo; *: significativo a $5 \% \mathrm{e}^{* *}$ : significativo a $1 \%$. 
Quanto à macroporosidade, esse resultado só foi semelhante para a camada superficial. Apesar desta situação ser característica do estado de compactação, o que pode ser confirmado pelo aumento na densidade do solo, a média dos valores de macroporosidade nas áreas com plantio direto nesta camada foi a mesma preconizada por Thomasson (1978) e Gupta \& Allmaras (1987) como mínima para o crescimento e satisfatória para as plantas, ou seja, $0,10 \mathrm{~m}^{3} \mathrm{~m}^{-3}$.

Maiores valores de densidade do solo no sistema plantio direto são normalmente encontrados em estudos deste tipo, pois neste sistema, apesar do revolvimento do solo ser restrito à linha de semeadura, o tráfego de máquinas ocorre normalmente em toda extensão da área, resultando num aumento da densidade nas camadas superficiais do solo (Assis \& Lanças, 2005).

A comparação entre sistema plantio direto de 12 anos irrigado com plantio direto de cinco anos mostrou que somente a macroporosidade, na camada $0-0,10 \mathrm{~m}$, apresentou diferença significativa, destacando-se o PD5, que apresentou maior valor. Observou-se também que o valor de macroporosidade no PD12 ficou abaixo do valor ideal para o crescimento e desenvolvimento das culturas.

A utilização da integração lavoura-pecuária proporcionou maiores valores de macroporosidade $\left(0,076-0,117 \mathrm{~m}^{3} \mathrm{~m}^{-3}\right)$ e porosidade total $(0,561$ $\left.0,611 \mathrm{~m}^{3} \mathrm{~m}^{-3}\right)$ na camada $0,10-0,20 \mathrm{~m}$, e ainda maior valor de microporosidade $\left(0,461-0,498 \mathrm{~m}^{3} \mathrm{~m}^{-3}\right) \mathrm{na}$ camada $0,20-0,30 \mathrm{~m}$. Esperava-se que a utilização da braquiária no sistema de produção alterasse as características físicas do solo, principalmente devido aos benefícios gerados pelo sistema radicular (Albuquerque et al., 2001). No entanto, devido à recente introdução deste sistema na área, os resultados benéficos podem ainda não ter se expressado.

Entre as camadas, as diferenças estatísticas somente ocorreram na área com plantio convencional. $\mathrm{Na}$ camada superficial, foram encontrados menor densidade, confirmada pelos maiores volumes de microporos e maiores de macroporos, reflexo da ação do equipamento utilizado no revolvimento e preparo do solo, que fraciona os agregados, com consequente aumento da porosidade.

Em todas as áreas avaliadas, os volumes de macroporos ficaram aquém do considerado ideal por Taylor \& Aschroft (1972), de aproximadamente $0,33 \mathrm{~m}^{3} \mathrm{~m}^{-3}$. Esse problema foi mais acentuado nas áreas sob sistema plantio direto $\left(0,09 \mathrm{~m}^{3} \mathrm{~m}^{-3}\right)$ e preparo convencional $\left(0,08 \mathrm{~m}^{3} \mathrm{~m}^{-3}\right)$. Esses dados indicam possíveis problemas de infiltração de água, circulação de oxigênio e, em consequência, crescimento das raízes das plantas (Thomasson, 1978; Secco et al., 2004).

\section{Índice $\mathrm{S}$}

Os valores de índice $\mathrm{S}$ em todas as áreas e profundidades ficaram acima de 0,035 , preconizado por
Dexter (2004a) como limite crítico para a boa qualidade do solo (Figura 2). Angelotti Netto et al. (2007) e Marchão et al. (2007) também encontraram variação para o índice S em função do sistema de uso e manejo. Assim como em seus estudos, o valor do $\mathrm{S}$ foi sempre superior àquele indicado por Dexter (2004a).

$\mathrm{O}$ sistema plantio direto mostrou-se com menor valor de índice $\mathrm{S}$ em relação ao plantio convencional na camada $0-0,10 \mathrm{~m}$ e maior na camada $0,20-0,30 \mathrm{~m}$. Esse resultado é um reflexo dos maiores valores de densidade do solo nesta camada no sistema plantio direto (Quadro 4). A área com sistema plantio direto de cinco anos, na camada $0,20-0,30 \mathrm{~m}$, apresentou maior valor de $\mathrm{S}$ do que a área que há 12 anos é irrigada. A integração lavoura-pecuária promoveu incremento no valor de S na camada mais profunda.

Considerando em conjunto as três camadas amostradas, o índice $\mathrm{S}$ correlacionou-se significativamente com todos os atributos físicos do solo avaliados e a MO (Figura 3), e as funções de regressão entre os atributos físicos em função de $\mathrm{S}$ ajustaram-se ao modelo linear. Assim, com o aumento da macroporosidade e o teor de MO, houve correlação positiva em relação ao índice S. Com o aumento da microporosidade e da densidade do solo, correlações negativas foram observadas, concordando com Stone et al. (2005) e Dexter (2004a). Verificou-se que o maior coeficiente de determinação foi encontrado para a macroporosidade, corroborando com Stone et al. (2005). Considerando o limite estabelecido por Dexter (2004a), $\mathrm{S} \geq 0$,035, para

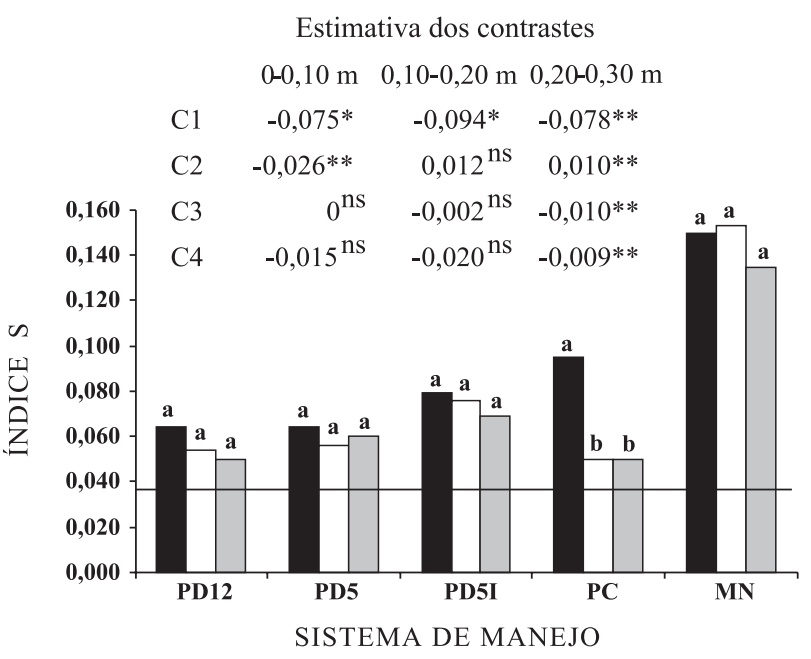

Figura 2. Valores de Índice $\mathrm{S}$ e estimativa dos contrastes estabelecidos para os cinco tratamentos e três profundidades. Camada de 0-0,10m ( $\square$ ); 0,10-0,20m ( $\square$ ); 0,20-0,30m ( $\square$ ); ns: não-significativo; *: significativo a $5 \% \mathrm{e} * *$ : significativo a $1 \%$. Médias seguidas pela mesma letra, dentro de cada manejo, não diferem estatisticamente entre si pelo teste de Tukey $(\mathrm{p}<0,05)$. 

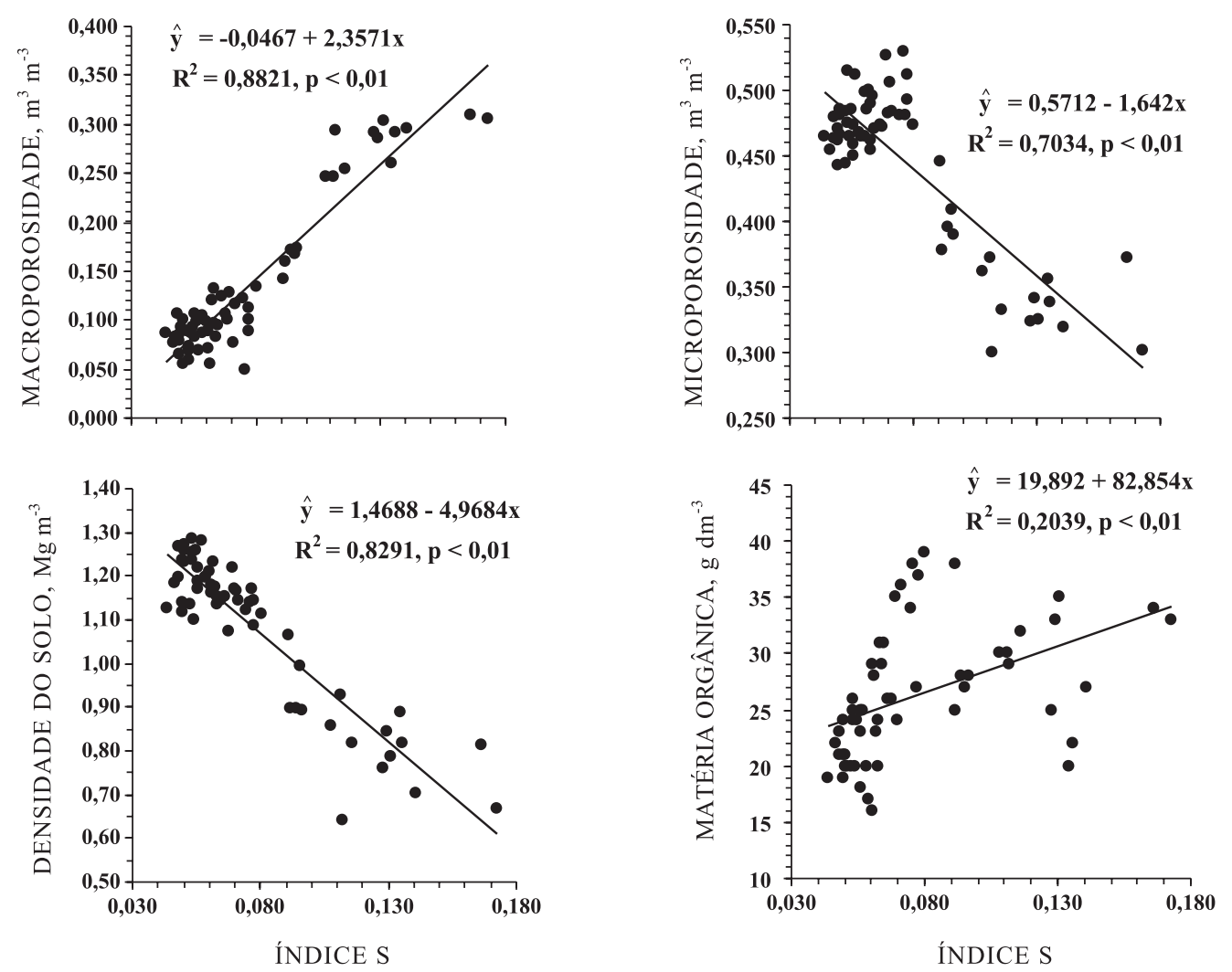

Figura 3. Regressão entre algumas propriedades físicas e matéria orgânica do Latossolo Vermelho acriférrico, em função do índice $S(n=60)$.

solos com boa qualidade estrutural, os valores limites para os atributos físicos do Latossolo Vermelho acriférrico seriam: macroporosidade $=0,026 \mathrm{~m}^{3} \mathrm{~m}^{-3}$, microporosidade $=0,513 \mathrm{~m}^{3} \mathrm{~m}^{-3}, \mathrm{Ds}=1,29 \mathrm{Mg} \mathrm{m}^{-3} \mathrm{eMO}$ $=17,83 \mathrm{~g} \mathrm{dm}^{-3}$. Observou-se que a macroporosidade estimada pela equação, utilizando o valor de 0,035 de $\mathrm{S}$, foi abaixo de $0,10 \mathrm{~m}^{3} \mathrm{~m}^{-3}$, considerada mínima por Thomasson (1978) e Secco et al. (2004) para o crescimento e desenvolvimento satisfatório das plantas.

\section{Análise multivariada}

A representação gráfica e os índices de correlação das variáveis nos componentes principais permitiu caracterizar as variáveis que mais influenciaram na discriminação dos grupos I, II, III e IV (Figura 4 e Quadro 4). As correlações com valores negativos no $\mathrm{CP} 1$, ou seja, à esquerda no gráfico, indicam que a RP $(-0,893)$ e a microporosidade $(-0,618)$ foram responsáveis pela discriminação dos grupos I e II, enquanto o índice $\mathrm{S}(0,967)$, a macroporosidade $(0,929)$, a Kfs $(0,833)$ e a porosidade total $(0,772)$ foram responsáveis pela formação dos grupos III e IV. Logo, pode-se inferir que as melhores condições estruturais do solo obtidas na área de mata (Grupo IV) permitiram a diferenciação desta em relação ao plantio direto (Grupos I e II). O preparo convencional apresentouse em posição intermediária.
Com relação ao segundo componente principal (CP2), a microporosidade (-0,628) discriminou o grupo III, representado pela área com preparo convencional do solo, plotada na porção inferior do eixo do CP2. Nesse caso, a menor perturbação do solo no sistema plantio direto e o excessivo revolvimento no preparo convencional influenciaram no aumento da densidade do solo, confirmado pela diminuição da microporosidade, apesar de alguns trabalhos evidenciarem que a microporosidade é pouco influenciada pelos efeitos do manejo do solo (Silva \& Kay, 1997; Araújo et al., 2004). Ainda no CP2, os índices de agregação do solo (DMG, DMP e Agreg) discriminaram os grupos I, II e IV, situados acima do eixo do CP2 no gráfico da figura 4. Esta resposta mostra que as áreas de plantio direto apresentam condições estruturais de agregação do solo próximas à área de mata. Vários trabalhos encontraram melhores índices de agregação na camada superficial nas áreas de plantio direto em relação ao preparo convencional (Campos et al., 1995; Silva et al., 2000; Wendling et al., 2005) e os autores relacionam tal comportamento ao incremento no teor de matéria orgânica. Os resultados deste trabalho sugerem que outros fatores inerentes aos sistemas ou ao solo podem estar atuando na agregação, já que não houve incremento de MO no sistema plantio direto, considerando o tempo de adoção avaliado (Figura 1). 


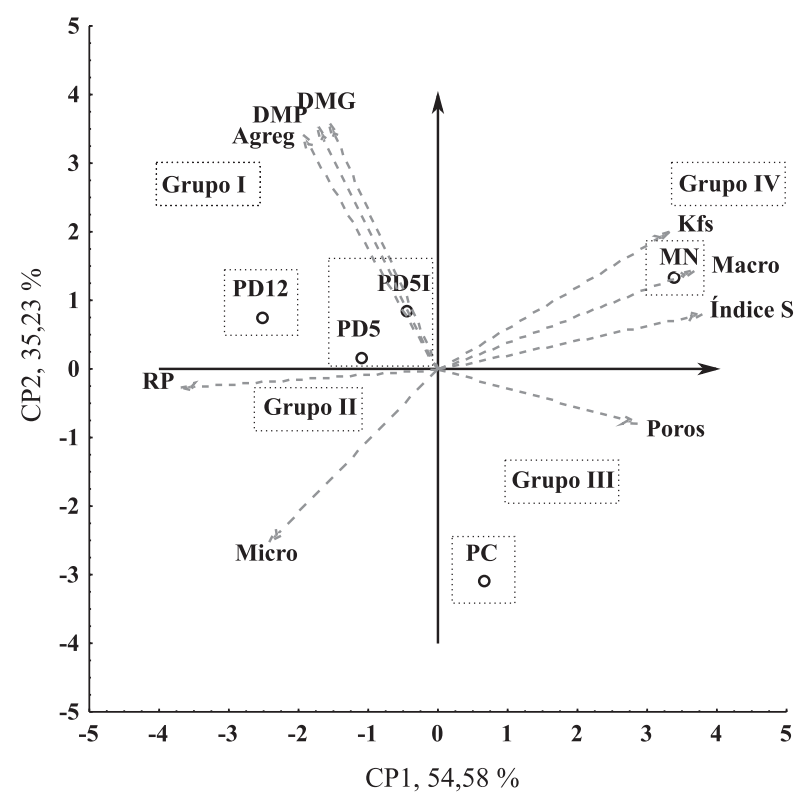

Figura 4. Dispersão (gráfico biplot) produzida por análise de componentes principais na profundidade $0-0,20 \mathrm{~m}$ das propriedades físicas do Latossolo Vermelho acriférrico típico (LVwf) submetidos a diferentes usos e manejos (PD12, PD5, PD5I, PC e MN): agreg = agregados com diâmetro $>2$ mm, DMG: diâmetro médio geométrico, DMP: diâmetro médio ponderado, macro: macroporosidade, micro: microporosidade, poros: porosidade total, RP: resistência do solo à penetração, e Kfs: condutividade hidráulica saturada.

Quadro 4. Correlação entre cada componente principal e as propriedades fisicas do LVwf submetidos a diferentes usos e manejos, para a camada de $0-0,20 \mathrm{~m}$

\begin{tabular}{lcc}
\hline Propriedade $^{(1)}$ & CP1 $^{(2)}$ & CP2 \\
\hline Agreg & $-0,505$ & 0,858 \\
DMG & $-0,425$ & 0,883 \\
DMP & $-0,450$ & 0,873 \\
Índice S & 0,967 & 0,173 \\
Poros & 0,772 & $-0,241$ \\
Macro & 0,929 & 0,365 \\
Micro & $-0,618$ & $-0,628$ \\
RP & $-0,893$ & $-0,068$ \\
Kfs & 0,833 & 0,518
\end{tabular}

(1) agreg: porcentagem de agregados com diâmetro $>2 \mathrm{~mm}$, DMG: diâmetro médio geométrico, DMP: diâmetro médio ponderado, poros: porosidade total, macro: macroporosidade, micro: microporosidade, RP: resistência do solo à penetração, Kfs: condutividade hidráulica saturada. ${ }^{(2)} \mathrm{CP} 1$ : componente principal 1; CP2: componente principal 2.

\section{CONCLUSÕES}

1. A área de mata nativa apresentou melhor qualidade física do solo, quando comparada às áreas submetidos à ação antrópica.

2. O sistema plantio direto não causou aumento do teor de matéria orgânica, porém causou maiores índices de agregação do que o plantio convencional.

3. A utilização da integração lavoura-pecuária, no seu primeiro ano de inclusão no sistema, não melhorou a qualidade física do solo.

4. O índice $\mathrm{S}$ mostrou variação entre os sistemas e foi sempre superior ao valor considerado limite para a boa qualidade física do solo.

\section{AGRADECIMENTOS}

À Fundação de Amparo à Pesquisa do Estado de São Paulo pela concessão de bolsa de estudo e auxílio à pesquisa para o desenvolvimento do projeto. Aos agricultores Fernando de Oliveira Carvalho e Rafael Takahashi, ambos de Guaíra (SP), proprietários das áreas onde se desenvolveu o projeto.

\section{LITERATURA CITADA}

ALBUQUERQUE, J.A.; SANGOI, L. \& ENDER M. Efeitos da integração lavoura-pecuária nas propriedades físicas do solo e características da cultura do milho. R. Bras. Ci. Solo, 25:717-723, 2001.

ANGELOTTI NETTO, A.; FERNANDES, J.E. \& CENTURION, J.F. Propriedades físicas e indicador de qualidade de um Latossolo Vermelho submetido a diferentes sistemas de uso e manejo. Irriga, 12:27-37, 2007.

ARAUJO, M.A.; TORMENA, C.A. \& SILVA, A.P. Propriedades físicas de um Latossolo Vermelho distrófico cultivado e sob mata nativa. R. Bras. Ci. Solo, 28:337-345, 2004.

ARSHAD, M. A.; LOWERY, B. \& GROSSMAN, B. Physical tests for monitoring soil quality. In: DORAN. J.W. \& JONES, A.J., eds. Methods for assessing soil quality. Madison, Soil Science Society of America, 1996. p.123-141.

ASSIS, R.L. \& LANÇAS, K.P. Avaliação dos atributos físicos de um Nitossolo Vermelho distroférrico sob sistema plantio direto, preparo convencional e mata nativa. R. Bras. Ci. Solo, 29:515-522, 2005.

BERTOL, I.; ALBUQUERQUE, J.A.; LEITE, D.; AMARAL, A.J. \& ZOLDAN JUNIOR, W.A. Propriedades físicas do solo sob preparo convencional e semeadura direta em rotação e sucessão de culturas, comparadas às do campo nativo. R. Bras. Ci. Solo, 28:155-163, 2004. 
BLAKE, G.R. \& HARTGE, K.H. Particle density. In: KLUTE, A, ed. Methods of soil analysis: Physical and mineralogical methods. 2.ed. Madison, American Society of Agronomy, 1986. p.377-382.

BOLINDER, M.A.; ANGERS, D.A.; GREEGORICH, E.G. \& CARTER, M.R. The response of soil quality indicators to conservation management. Can. J. Soil Sci., 79:37-45, 1999.

CARTER, M.R.; GREGORICH, E.G.; ANDERSO, D.W.; DORAN, J.W.; JANZEN, H.H. \& PIERCE, F.J. Concepts of soil quality and their significance. In: GREGORICH, E.G. \& CARTER, M.R., eds. Soil quality for crop production and ecosystem health. Amsterdam, Elservier Science, 1997. p.1-20.

CAMPOS, B.C.; REINERT, D.J.; NICOLODI. R,; RUEDELL, J. \& PETRERE, C. Estabilidade estrutural de um Latossolo Vermelho-Escuro distrófico após sete anos de rotação de culturas e sistemas de manejo de solo. R. Bras. Ci. Solo, 19:121-126, 1995.

CASTRO FILHO, C.; MUZILli, O. \& PODANOSCHI, A.L. Estabilidade de agregados e sua relação com o teor de carbono orgânico num Latossolo Roxo distrófico, em função de sistemas de plantio, rotação de culturas e métodos de preparo das amostras. R. Bras. Ci. Solo, 22:527538, 1998.

CAVENAGE, A.; MORAES, M.L.T.; ALVES, M.C.; CARVALHO, M.A.C.; FREITAS, M.L.M. \& BUZETTI, S. Alterações nas propriedades físicas de um Latossolo Vermelho-Escuro sob diferentes culturas. R. Bras. Ci. Solo, 23:997-1003, 1999.

CORAZZA, E.J.; SILVA, J.E.; RESCK, D.V.S. \& GOMES, A.C. Comportamento de diferentes sistemas de manejo como fonte ou depósito de carbono em relação à vegetação de Cerrado. R. Bras. Ci. Solo, 23:425-432, 1999.

DANIELSON, R.E. \& SUTHERLAND, P.L. Porosity. In: KLUTE, A., ed. Methods of soil analysis. 2.ed. Madison, American Society of Agronomy, 1986. Part 1. p.443-461.

DEXTER, A.R. Soil physical quality: Part I. Theory. Effects of soil texture, density, and organic matter, and effects on root growth. Geoderma, 120:201-214, 2004a.

DEXTER, A.R. Soil physical quality: Part II. Friability, tillage, tilth and hard-setting. Geoderma, 120:215-225, 2004b.

DEXTER, A.R. Soil physical quality: Part III. Unsaturated hydraulic conductivity and general conclusions about Stheory. Geoderma, 120:227-239, 2004c.

DORAN, J.W.; SARRANTONIO, M. \& LIEBIG, M.A. Soil health and sustainability. Adv. Agron., 56:1-54, 1996.

EMPRESA BRASILEIRA DE PESQUISA AGROPECUÁRIA EMBRAPA. Sistema brasileiro de classificação de solos. Rio de Janeiro, Embrapa Solos, 2006. 306p.

EMPRESA BRASILEIRA DE PESQUISA AGROPECUÁRIA EMBRAPA. Sistema Santa Fé - Tecnologia Embrapa: Integração lavoura pecuária pelo consórcio de culturas anuais com forrageiras, em áreas de lavoura, nos sistemas plantio direto e convencional. Santo Antônio de Goiás, Embrapa Arroz e Feijão, 2000. 28p.
GREGORICH, E.G. Quality. In: LAL, R., ed. Encyclopedia of soil science. New York, Marcel Dekker, 2002. p.10581061.

GUPTA, S.C. \& ALLMARAS, R.R. Models to assess the susceptibility of soils to excessive compaction. Adv. Soil Sci., 6:65-100, 1987.

HORN, R.; DOMZAL, H.; SLOWINSKA-JURKIEWICZ, A. \& OUWERKERK, C. Soil compaction processes and their effects on the structure of arable soils and the enviroment. Soil Till. Res., 35:23-36, 1995.

KAISER, H.F. The varimax criterion for analytic rotation in factor analysis. Psychometrika, 23:187-200, 1958.

KEMPER, W.D. \& CHEPIL, W.S. Aggregate stability and size distribution. In: BLACK, C. A., ed. Methods of soil analysis. Madison, ASA, 1965. Part 1. p.499-510.

KLEPKER, D. \& ANGHINONI, I. Características físicas e químicas do solo afetadas por métodos de preparo e modos de adubação. R. Bras. Ci. Solo, 19:395-401, 1995.

KLUTE, A. Water retention: Laboratory methods. In: KLUTE, A., ed. Methods of soil analysis. 2.ed. Madison, American Society of Agronomy, 1986. Part. 1. p.635-662.

MARCHÃO, R.L.; SANTOS JUNIOR, J.D.G.; SILVA, E.M.; SÁ, M.A.C.; BALBINO, L.C.; VILELA, L. \& BECQUER, T. Parâmetro "S" e intervalo hídrico ótimo em Latossolo Vermelho sob sistemas de integração lavoura-pecuária no Cerrado. In: CONGRESSO BRASILEIRO DE CIÊNCIA DO SOLO, 31., 2007. Anais. Gramado, Sociedade Brasileira de Ciência do solo, 2007. CD-ROM.

RAIJ, B.van; QUAGGIO, J.A.; CANTARELLA, H.; FERREIRA, M.; LOPES, A.S. \& BATAGLIA, O.C. Análise química do solo para fins de fertilidade. Campinas, Fundação Cargill, 1987. $170 \mathrm{p}$.

REYNOLDS, W.D.; VIEIRA, S.R. \& TOPP, G.C. An assessment of the single-head analysis for the Constant head well permeameter. Can. J. Soil Sci., 72:489-501, 1992.

SECCO, D.; REINERT, D.J.; REICHERT, J.M. \& DA ROS, C.O. Produtividade de soja e propriedades físicas de um Latossolo submetido a sistemas de manejo e compactação. R. Bras. Ci. Solo, 28:797-804, 2004.

SILVA, A.P. \& KAY, B.D. The sensitivity of shoot growth of corn to the least limiting water range of soils. Plant Soil, 184:323-329, 1996.

SILVA, A.P. \& KAY, B.D. Estimating the least limiting water range of soils from properties and management. Soil Sci. Soc. Am. J., 61:877-883, 1997.

SILVA, A.P. Integração manejo $x$ física. In: REUNIÃO BRASILEIRA DE MANEJO E CONSERVAÇÃO DO SOLO E DA ÁGUA, 15., Santa Maria, 2004. Palestras... Santa Maria, UFSM, 2004. CD-ROM.

SILVA, M.L.N.; CURI, N. \& BLANCANEAUX, P. Sistemas de manejo e qualidade estrutural de um Latossolo Roxo. Pesq. Agropec. Bras., 35:2485-2492, 2000.

STATSOFT, INC. Statistica for Windows - Computer program manual. Tulsa, 1995. 
STOLF, R.; FERNANDES, J. \& FURLANI NETO, V.L. Penetrômetro de impacto IAA/PLANALSUCAR-STOLF: Recomendação para seu uso. STAB, 3:18-23, 1983.

STONE, L.F.; BALBINO, L.C. \& CUNHA, E.Q. Índice S como indicador da qualidade física do solo. In: CONGRESSO BRASILEIRO DE ENGENHARIA AGRÍCOLA, 34. Canoas, 2005. Resumos. Canoas, Sociedade Brasileira Engenharia Agrícola, 2005. CD-ROM.

TAYLOR, S.A. \& ASHCROFT, G.L. Physical edaphology The physics of irrigated and nonirrigated soils. San Francisco, W. H. Freeman, 1972. 532p.

THOMASSON, A.J. Towards an objective classification of soil structure. J. Soil Sci., 29:38-46, 1978.
TORMENA, C.A.; VIDIGAL FILHO, P.S.; GONÇALVES, A.C.A.; ARAUJO, M.A. \& PINTRO, J.C. Influência de diferentes sistemas de preparo do solo nas propriedades físicas de um Latossolo Vermelho distrófico. R. Bras. Eng. Agríc. Amb., 8:65-71, 2004.

van GENUCHTEN, M.T.A. A closed-form equation for predicting the hydraulic conductivity of unsaturated soils. Soil Sci. Soc. Am. J., 44:892-897, 1980

WENDLING, B.; JUCKSCH, I.; MENDONCA, E.S \& NEVES, J.C.L. Carbono orgânico e estabilidade de agregados de um Latossolo Vermelho sob diferentes manejos. Pesq. Agropec. Bras., 40:487-494, 2005.

YOODER, R.E. A direct methodo of aggregate analysis of soils and a study of the physical nature of erosion losses. J. Am. Soc. Agric., 28:337-351, 1936. 
